\title{
Effect of oral tranexamic acid on macular edema associated with retinal vein occlusion or diabetes
}

This article was published in the following Dove Press journal:

Clinical Ophthalmology

\author{
Masayuki Takeyama' \\ Fumio Takeuchi ${ }^{2}$ \\ Masahiko Gosho 3 \\ Keijiro Sugita \\ Masahiro Zako ${ }^{4}$ \\ Masayoshi Iwaki ${ }^{5}$ \\ Motohiro Kamei' \\ 'Department of Ophthalmology, \\ Aichi Medical University, \\ Nagakute, ${ }^{2}$ Department of \\ Biochemistry, Aichi Medical University, \\ Nagakute, ${ }^{3}$ Department of Clinical \\ Trial and Clinical Epidemiology, \\ Faculty of Medicine, University of \\ Tsukuba, Tsukuba, ${ }^{4}$ Department of \\ Ophthalmology, Asia Hospital, Seto, \\ ${ }^{5}$ Department of Ophthalmology, \\ Yokkaichi, Digestive Disease Center, \\ Komono, Japan
}

\begin{abstract}
Purpose: Tranexamic acid (TXA) is a widely used antifibrinolytic agent that can also cause a decrease in vascular permeability. We hypothesized that TXA could improve macular edema (ME) that is caused by an increase in retinal vascular permeability. The aim of this study is to evaluate the efficacy of oral TXA for ME associated with retinal vein occlusion (RVO) or diabetic ME (DME).

Patients and methods: Oral TXA (1,500 mg daily for 2 weeks) was administered to patients with persistent ME secondary to RVO (7 eyes) and DME ( 7 eyes). After 2 weeks (ie, the final day of administration) and 6 weeks (ie, 4 weeks after the final administration), best-corrected visual acuity and central macular thickness (CMT) were measured and compared with baseline. Analyses were performed for RVO and DME cases. No other treatment was performed during the study period.
\end{abstract}

Results: In RVO cases, significant improvement in CMT was found between baseline $(467.7 \pm 121.4 \mu \mathrm{m})$ and 2-week measurements after treatment (428.7 $\pm 110.5 \mu \mathrm{m}, p=0.024)$. No significant change was found in CMT between measurements taken at baseline and 6 weeks after treatment. In DME cases, no significant change was found in CMT between measurements taken at baseline and 2 or 6 weeks after treatment. In all analyses of best-corrected visual acuity, no significant change was observed.

Conclusion: The results support the hypothesis that plasmin plays a role in the development of ME associated with RVO, and oral TXA administration may be useful as an adjuvant treatment when combined with other agents such as anti-vascular endothelial growth factor.

Keywords: diabetic macular edema, fibrin, macular edema, plasmin, retinal vein occlusion, tranexamic acid

\section{Introduction}

Macular edema (ME) is a major cause of visual impairment in patients with retinal vascular disorders, such as retinal vein occlusion (RVO) and diabetic ME (DME). ${ }^{1}$ Although the pathogenesis of ME is complex, the most direct mechanism is the disruption of the blood-retinal barrier. Because vascular endothelial growth factor (VEGF) is believed to play a key role in the disruption of the blood-retinal barrier, the use of intravitreal injections of anti-VEGF agents to treat ME has been increasing during the last decade. ${ }^{2}$ Anti-VEGF therapy is regarded as the first-line treatment in a majority of ME cases, and numerous studies have demonstrated its efficacy. However, there is a risk of severe complications, including infectious endophthalmitis and retinal detachment, arising from the use of intravitreal injection, although these are relatively rare. ${ }^{3,4}$ Systemic side effects, including an increase in thromboembolic events $^{5,6}$ and kidney toxicity, ${ }^{7,8}$ have also been reported. Although ocular side effects have not yet been clearly defined, some unfavorable phenomena have been reported,
Correspondence: Masayuki Takeyama Department of Ophthalmology, Aichi Medical University, I-I, Yazakokarimata Nagakute-shi, Aichi, 480-I I95, Japan

Tel +8I 56 I62 33। I

Fax +8I $56 I 637255$

Email mtakeyam@gmail.com 
including choroidal thinning, retinal pigment epithelium tear, uveitis, retinal venous occlusion, retinal artery occlusion, and ocular hypertension. ${ }^{9-11}$ In addition, prolonged and extreme inhibition of VEGF, which is induced by repeated injections, may interfere with the physiological function of the retina. ${ }^{12}$ Moreover, commercially available anti-VEGF agents are expensive and so have important social implications. ${ }^{13}$ Given the concerns surrounding the use of intravitreal injection of anti-VEGF agents, alternative treatments and methods to decrease the frequency of injection require investigation.

Plasmin, which is converted from plasminogen by plasminogen activator, is known as the principal enzyme in the fibrinolytic system and is recognized for its many nonfibrinolytic activities, including the promotion of vascular permeability and neuronal injury. ${ }^{14}$ Previous reports have demonstrated that plasmin can disrupt the blood-brain barrier and contribute to the development of brain edema. ${ }^{14,15}$ The known pathways through which plasmin promotes vascular permeability include the following: activation of matrix metalloproteinases, which are the principal regulators of vascular permeability; ${ }^{16,17}$ degradation of vascular basement membrane components, including collagen IV, laminin, and fibronectin; ${ }^{14,18}$ activation of platelet-derived growth factor-C pathway; ${ }^{14}$ promotion of an inflammatory response, ${ }^{19-23}$ and contraction ${ }^{24,25}$ or apoptosis ${ }^{26}$ of vascular endothelial cells. Furthermore, plasmin acts on both intravascular and extravascular tissues. In the brain, excessive plasmin activity, often induced by ischemia, injures neurons. ${ }^{27-29}$ Taken together, plasmin can injure both the vascular structures and the whole neurovascular unit. In accordance with the concept of a common neurovascular unit between the brain and retina, we suspected that plasmin may contribute to the development of ME and retinal cell damage secondary to retinal vascular disease.

Tranexamic acid (TXA) is a widely used antifibrinolytic agent that was discovered in the $1960 \mathrm{~s} .{ }^{30}$ It binds to lysinebinding sites on plasminogen and subsequently causes decreased activation of plasminogen to plasmin. ${ }^{31}$ It has been clinically demonstrated to induce hemostasis, reduce inflammation, and decrease vascular permeability. As a hemostatic agent, clinical indications for TXA are hyphema, menorrhagia, trauma, and perioperative use. ${ }^{31}$ In addition, TXA has been prescribed for the treatment of pharyngitis, laryngitis, tonsillitis, ${ }^{32,33}$ and angioedema ${ }^{34,35}$ owing to its ability to reduce inflammation and vascular permeability.

Given its reported efficacy in the reduction of vascular permeability, we hypothesized that TXA could improve $\mathrm{ME}$ and damaged retinal function associated with retinal vascular disease. Because the efficacy, safety, and tolerability of TXA were established by previous clinical studies, ${ }^{36,37}$ we administrated oral TXA for the treatment of ME associated with RVO or diabetes.

\section{Patients and methods}

This study is a prospective, interventional case series of 14 eyes in 12 patients with ME secondary to RVO (including branch RVO [BRVO] and central RVO [CRVO]) and DME. The study protocol was approved by the institutional review board of Aichi Medical University Hospital, Nagakute, Japan. This study was registered at the University Hospital Medical Information Network (study ID: UMIN 000014601).

Inclusion criteria were as follows: 1) central macular thickness (CMT, average retinal thickness within a $1 \mathrm{~mm}$ diameter circle centered on the fovea) exceeding $300 \mu \mathrm{m}$ when measured with spectral domain optical coherence tomography (RS-3000, Nidek, Aichi, Japan); 2) the presence of chronic edema for $\geq 6$ months; 3 ) history of persistent ME despite previous treatment, including intravitreal injection of an antiVEGF agent, sub-Tenon injection of steroid, vitrectomy, or laser photocoagulation. Exclusion criteria were as follows: 1) optical coherence tomography image showing vitreomacular traction or thick epiretinal membrane; 2) inappropriate general physical condition, kidney failure (blood creatinine concentration exceeding $1.5 \mathrm{mg} / \mathrm{dL}$ or artificial dialysis), uncontrolled hypertension (systolic and diastolic blood pressures exceeding 160 and $90 \mathrm{mmHg}$, respectively), or severe hyperglycemia in patients with diabetes (glycated hemoglobin values $>9.0 \%$ ); 3) any treatment for ME within the last 3 months; 4) severe impairment in macular function, best-corrected visual acuity $($ BCVA) $>1.0$ (logarithm of minimum angle of resolution); and 5) fluorescein angiogram suggesting involvement of aneurysms near the fovea in ME development. All patients were informed of the off-label use of TXA, and they provided written informed consent for this study.

Before beginning TXA administration, all the patients underwent complete ocular examination, including measurement of the BCVA and intraocular pressure, slit-lamp and fundus examination, fundus photography, and CMT measurement. TXA (Transamin ${ }^{\circledR}$ capsule, Daiichi Sankyo Co. Ltd., Tokyo, Japan) was prescribed for oral administration at a dosage of $500 \mathrm{mg}$ thrice daily to achieve a total dosage of 1,500 mg per day, for 2 weeks. After 2 weeks (ie, the final day of administration) and 6 weeks (ie, 4 weeks after the final administration), BCVA and CMT were measured and assessed as the main outcomes. No other treatment was performed during the study period. 
BCVA and CMT at 2 and 6 weeks were compared with those at baseline using one-way analysis of variance. The multiplicity of testing was adjusted using Dunnett's procedure with week 0 as control. Analyses were performed for RVO and DME cases because the mechanism for breaching the blood-retinal barrier is different in both. All statistical tests were 2 -sided with a 5\% significance level. All analyses were performed using the SAS software version 9.4 (SAS Institute, Cary, NC, USA).

\section{Results}

\section{RVO cases}

Seven eyes of 7 patients ( 6 men and 1 woman) were included. The patients' mean age was 70.1 (range 52-83) years. The mean duration of ME was 40.0 (range 11-90) months. Four BRVO eyes and 3 CRVO eyes were analyzed together as RVO cases. No statistically significant difference was found in BCVA between the baseline $(0.30 \pm 0.17)$ and the 2-week $(0.32 \pm 0.22, p=0.87)$ and 6-week $(0.37 \pm 0.23, p=0.58)$ measurements. For CMT, a statistically significant difference was found between the baseline $(467.7 \pm 121.4 \mu \mathrm{m})$ and 2-week (428.7 $\pm 110.5 \mu \mathrm{m}, p=0.024)$ measurements. However, no significant change was found between the baseline and 6-week ( $450.9 \pm 128.0 \mu \mathrm{m}, p=0.49)$ measurements. No systemic side effects were found during the study period. All RVO patients' baseline clinical characteristics and results are summarized in Table 1. A representative case of BRVO is shown in Figure 1.

\section{DME cases}

Seven eyes of 5 patients (all women) were included. The patients' mean age was 63.0 (range 47-74) years. The mean duration of ME was 32.3 (range 14-44) months. For all 7 DME eyes, no statistically significant difference was found in BCVA between the baseline $(0.51 \pm 0.20)$ and the 2-week $(0.47 \pm 0.16, p=0.71)$ and 6-week $(0.53 \pm 0.27, p=0.96)$ measurements. For CMT, no statistically significant differences were found in the measurements taken at baseline $(551.3 \pm 164.8 \mu \mathrm{m})$ compared with those taken at 2 weeks (504.9 $\pm 151.3 \mu \mathrm{m}, p=0.12)$ and 6 weeks $(494.3 \pm 152.7 \mu \mathrm{m}$, $p=0.063)$. No systemic side effects were noted during the study period. All DME patients' baseline clinical characteristics and results are summarized in Table 2 .

\section{Discussion}

The current study demonstrated that administration of oral TXA could potentially reduce ME associated with RVO. Because this was a pilot study, we administered TXA for only

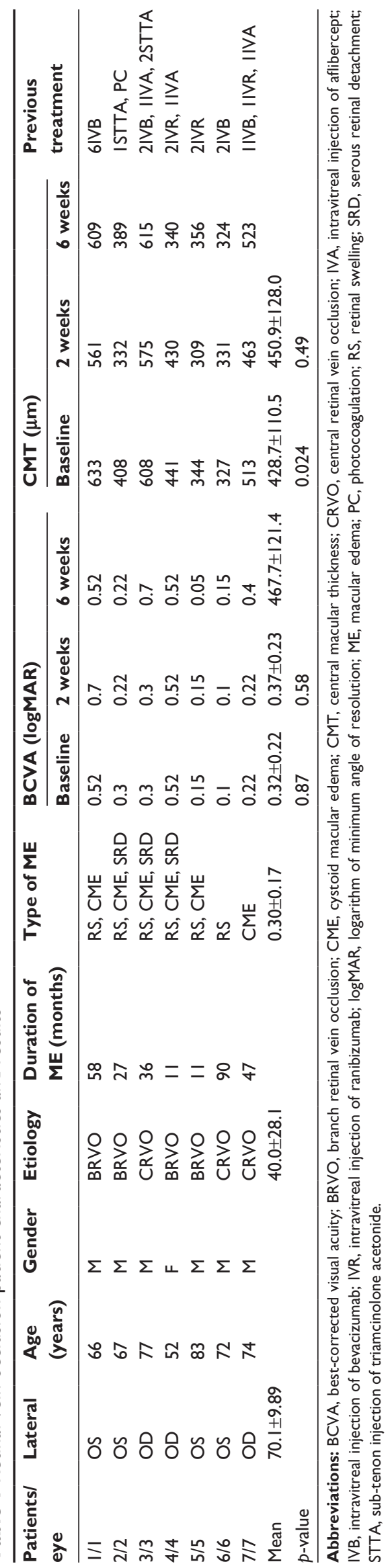




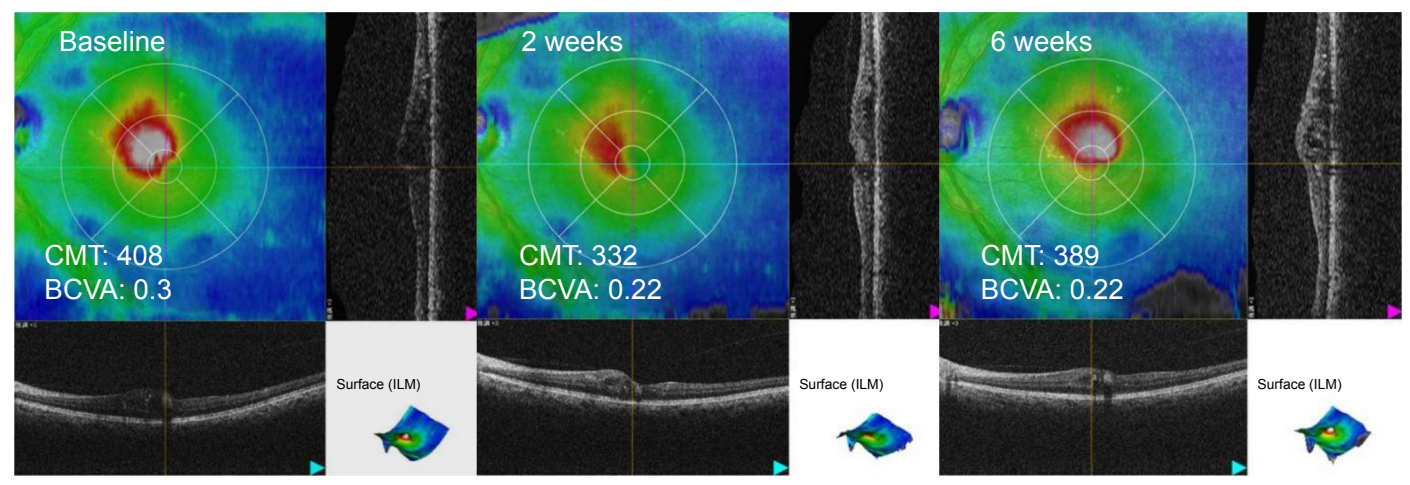

Figure I Optical coherence tomography scans in a case of branch retinal vein occlusion (eye 2).

Note: The scans demonstrate decreased macular edema after 2-week administration of tranexamic acid and the deterioration at the 6-week time point.

Abbreviations: BCVA, best-corrected visual acuity; CMT, central macular thickness ( $\mu \mathrm{m})$; ILM, internal limiting membrane; LogMAR, logarithm of minimum angle of resolution.

2 weeks, and yet we found a significant decrease in CMT in RVO cases 2 weeks after the treatment commenced. ME rebounded 4 weeks after stopping TXA treatment, suggesting that continuous dosing may suppress the edema for a longer duration, and the mechanism may not involve morphological changes, including remodeling the extracellular matrix. There may be several explanations as to why no significant decrease in CMT in DME patients was observed. The presence of ME in DME and RVO could be caused by different pathological mechanisms, or the pathological changes may be more severe in DME than RVO, and more time is required to remedy the condition. Generally, the efficacy of the 2-week intake of TXA for ME was limited.

A difference in CMT was found before and after the administration of TXA, which suggests that plasmin involvement in ME development associated with RVO is similar to that in brain edema. Therefore, plasmin may be a therapeutic target for the treatment of ME, although this protocol with a 2-week intake of TXA cannot sufficiently demonstrate this. In brain capillaries, ischemic injury damages tight-junction proteins and permits easier access of blood-derived plasmin to the basement membrane, creating a positive feedback loop. ${ }^{14}$ Plasmin probably also acts in retinal capillaries injured by ischemia. In addition, deposits of fibrin have been observed in the retinal capillaries of RVO patients. ${ }^{38-40}$ Fibrin deposits in the retinal capillaries continuously activate plasminogen, and the generated plasmin may injure the basement membrane. We speculate that plasmin is involved in the development of ME through these mechanisms, and that inhibition of fibrinolytic activity by TXA disrupts these mechanisms and decreases ME.

BCVA was unchanged in all analyses, possibly because of the latency in visual improvement when compared with morphological improvement. Discrepancy between visual acuity and CMT is often observed clinically, especially at the initial stage of the treatment, where CMT improvement occurs earlier and visual improvement is delayed by several weeks. Because we administered TXA for only 2 weeks in the present study, ME recurred before visual improvement was achieved. Longer dosing may lead to an improvement in visual acuity.

No side effects of TXA treatment were observed in this study. Although the safety of TXA had been established by previous clinical studies, ${ }^{36,37}$ an increased risk of thrombotic events should be discussed because patients with retinal vascular disease have increased risk of thrombosis. ${ }^{41}$ Many researchers have previously investigated whether TXA increases the risk of thrombosis, but came up with conflicting results. ${ }^{36,42,43}$ In a randomized controlled trial that included about 20,000 patients with traumatic hemorrhage, TXA appeared to decrease the risk of arterial thrombosis. ${ }^{44}$ This could be because of the anti-inflammatory properties of TXA. ${ }^{45}$ Therefore, the likelihood of TXA inducing a thrombotic event is considered to be low, despite its robust antifibrinolytic effect.

Previous studies have shown the associations of various types of ME, including retinal swelling, cystoid ME, and serous retinal detachment, with the treatment effect. For example, ME with serous retinal detachment is known to be relatively incurable with anti-VEGF agents. ${ }^{46-48}$ Although the results of our study did not suggest such a tendency, larger studies may reveal differences among these types of ME.

Our results showed that the effect of TXA on DME was not significant, unlike the effect on RVO. However, whether TXA is effective for DME cannot actually be determined. The grade of retinal ischemia observed on the fluorescein angiogram in all patients with RVO was mild (no patient had involvement of more than 5 optic disc areas). In contrast, patients with DME exhibited various grades of diabetic retinopathy. Although a comparison between RVO and DME is 


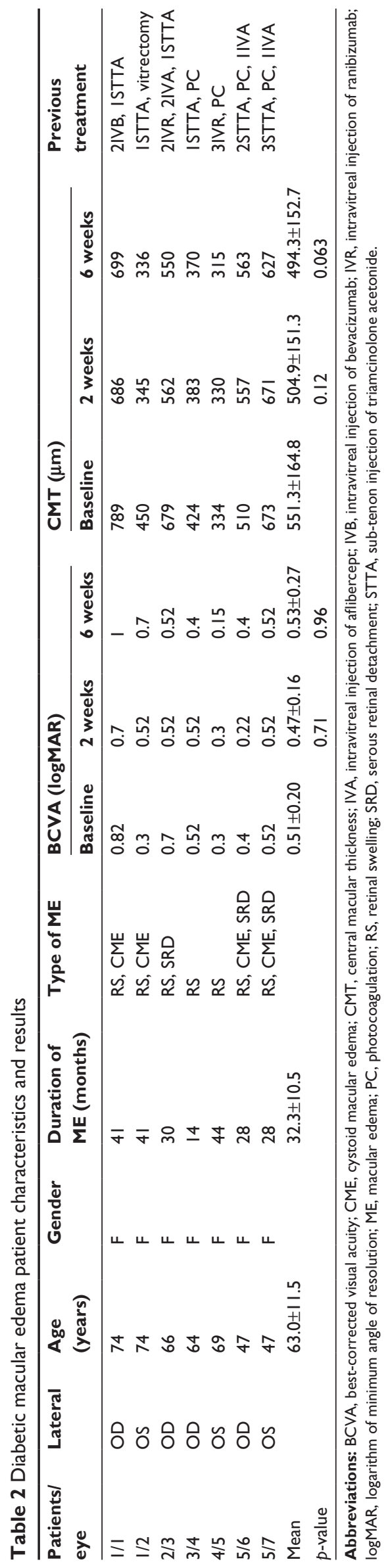

not possible, the degree of ischemia in the whole retina of patients with DME was generally more severe than that of those with RVO. Although we excluded patients with ischemic maculopathy, peripheral retinal ischemia can influence ME. ${ }^{49}$ In addition, the baseline CMT in patients with DME was considerably severe. These patient characteristics might have affected the outcome.

The major shortcomings of this pilot study are the small number of participants, heterogeneity among the participants, and the lack of a control group. A study with a larger number of participants or more rigidly designed criteria is needed.

\section{Conclusion}

The results of this pilot study suggest that plasmin plays a role in the development of ME associated with RVO and oral administration of TXA could be a useful adjuvant treatment when combined with other treatments such as anti-VEGF agents.

\section{Disclosure}

MG received lecture and/or consultation fees from Daiichi Sankyo Co., Ltd., Ferring Pharmaceuticals Co., Ltd, Novartis and Tiho Pharma Co., Ltd, travel fees from Takeda Pharmaceutical Co., Ltd, and manuscript fees from Kowa Co., Ltd. The authors report no other conflicts of interest in this work.

\section{References}

1. Tomkins-Netzer O, Ismetova F, Bar A, Seguin-Greenstein S, Kramer M, Lightman S. Functional outcome of macular edema in different retinal disorders. Prog Retin Eye Res. 2015;48:119-136.

2. Coscas G, Cunha-Vaz J, Soubrane G. Macular edema: definition and basic concepts. Dev Ophthalmol. 2017;58:1-10.

3. Inoue M, Kobayakawa S, Sotozono C, et al. Evaluation of the incidence of endophthalmitis after intravitreal injection of anti-vascular endothelial growth factor. Ophthalmologica. 2011;226(3):145-150.

4. Dossarps D, Bron AM, Koehrer P, Aho-Glélé LS, Creuzot-Garcher C; FRCR net (FRenCh Retina specialists net). Endophthalmitis after intravitreal injections: incidence, presentation, management, and visual outcome. Am J Ophthalmol. 2015;160(1):17-25.

5. Schlenker MB, Thiruchelvam D, Redelmeier DA. Intravitreal anti-vascular endothelial growth factor treatment and the risk of thromboembolism. Am J Ophthalmol. 2015;160(3):569-580.e5.

6. Tolentino M. Systemic and ocular safety of intravitreal anti-VEGF therapies for ocular neovascular disease. Surv Ophthalmol. 2011; 56(2):95-113.

7. Pellé G, Shweke N, Duong Van Huyen JP, et al. Systemic and kidney toxicity of intraocular administration of vascular endothelial growth factor inhibitors. Am J Kidney Dis. 2011;57(5):756-759.

8. Georgalas I, Papaconstantinou D, Papadopoulos K, Pagoulatos D, Karagiannis D, Koutsandrea C. Renal injury following intravitreal antiVEGF administration in diabetic patients with proliferative diabetic retinopathy and chronic kidney disease a possible side effect? Curr Drug Saf. 2014;9(2):156-158.

9. Branchini L, Regatieri C, Adhi M, et al. Effect of intravitreous antivascular endothelial growth factor therapy on choroidal thickness in neovascular age-related macular degeneration using spectral-domain optical coherence tomography. JAMA Ophthalmol. 2013;131(5): 693-694. 
10. van der Reis MI, La Heij EC, De Jong-Hesse Y, Ringens PJ, Hendrikse F, Schouten JS. A systematic review of the adverse events of intravitreal anti-vascular endothelial growth factor injections. Retina. 2013;31(8):1449-1469.

11. Falavarjani KG, Nguyen QD. Adverse events and complications associated with intravitreal injection of anti-VEGF agents: a review of literature. Eye (Lond). 2013;27(7):787-794.

12. Kurihara T, Westenskow PD, Bravo S, Aguilar E, Friedlander M. Targeted deletion of Vegfa in adult mice induces vision loss. J Clin Invest. 2012;122(11):4213-4217.

13. Kwong TQ, Mohamed M. Anti-vascular endothelial growth factor therapies in ophthalmology: current use, controversies and the future. Br J Clin Pharmacol. 2014;78(4):699-706.

14. Niego B, Medcalf RL. Plasmin-dependent modulation of the bloodbrain barrier: a major consideration during tPA-induced thrombolysis? J Cereb Blood Flow Metab. 2014;34(8):1283-1296.

15. Gingrich MB, Traynelis SF. Serine proteases and brain damage - is there a link? Trends Neurosci. 2000;23(9):399-407.

16. Monea S, Lehti K, Keski-Oja J, Mignatti P. Plasmin activates pro-matrix metalloproteinase- 2 with a membrane-type 1 matrix metalloproteinasedependent mechanism. J Cell Physiol. 2002;192(2):160-170.

17. Jin R, Yang G, Li G. Molecular insights and therapeutic targets for blood-brain barrier disruption in ischemic stroke: critical role of matrix metalloproteinases and tissue-type plasminogen activator. Neurobiol Dis. 2010;38(3):376-385.

18. Rabbani LE, Johnstone MT, Rudd MA, Devine P, George D, Loscalzo J. PPACK attenuates plasmin-induced changes in endothelial integrity. Thromb Res. 1993;70(6):425-436.

19. Laurberg G. Plasma kinin activation in tranexamic acid treated patients with hereditary angioneurotic edema. Arch Dermatol Res. 1978; 262(2):153-156.

20. Moroi M, Aoki N. Inhibition of proteases in coagulation, kinin-forming and complement systems by alpha2-plasmin inhibitor. J Biochem. 1977; 82(4):969-972.

21. Kleniewski J, Blankenship DT, Cardin AD, Donaldson V. Mechanism of enhanced kinin release from high molecular weight kininogen by plasma kallikrein after its exposure to plasmin. J Lab Clin Med. 1992; 120(1):129-139.

22. Yao Y, Tsirka SE. Truncation of monocyte chemoattractant protein 1 by plasmin promotes blood-brain barrier disruption. J Cell Sci. 2011; 124(Pt 9):1486-1495.

23. Miles LA, Parmer RJ. Plasminogen receptors: the first quarter century. Semin Thromb Hemost. 2013;39(4):329-337.

24. Nagy Z, Kolev K, Csonka E, Pék M, Machovich R. Contraction of human brain endothelial cells induced by thrombogenic and fibrinolytic factors. An in vitro cell culture model. Stroke. 1995;26(2):265-270.

25. Nagy Z, Kolev K, Csonka E, Vastag M, Machovich R. Perturbation of the integrity of the blood-brain barrier by fibrinolytic enzymes. Blood Coagul Fibrinolysis. 1998;9(6):471-478.

26. Doeuvre L, Plawinski L, Goux D, Vivien D, Anglés-Cano E. Plasmin on adherent cells: from microvesiculation to apoptosis. Biochem J. 2010;432(2):365-373.

27. Tsirka SE, Bugge TH, Degen JL, Strickland S. Neuronal death in the central nervous system demonstrates a non-fibrin substrate for plasmin. Proc Natl Acad Sci US A. 1997;94(18):9779-9781.

28. Junge CE, Sugawara T, Mannaioni G, et al. The contribution of protease-activated receptor 1 to neuronal damage caused by transient focal cerebral ischemia. Proc Natl Acad Sci U S A. 2003;100(22): 13019-13024.

29. Cunningham O, Campion S, Perry VH, et al. Microglia and the urokinase plasminogen activator receptor/uPA system in innate brain inflammation. Glia. 2009;57(16):1802-1814.

30. Okamoto S, Sato S, Takada Y, Okamoto U. An active stereo-isomer (trans-form) of AMCHA and its antifibrinolytic (antiplasmic) action in vitro and in vivo. Keio J Med. 1964;13:177-185.
31. Tengborn L, Blombäck M, Berntorp E. Tranexamic acid-an old drug still going strong and making a revival. Thromb Res. 2015;135(2): 231-242.

32. McCormack PL. Tranexamic acid: a review of its use in the treatment of hyperfibrinolysis. Drugs. 2012;72(5):585-617.

33. Camarri E, Amerighi A, Bellofiore $P$, et al. The clinical use of tranexamic acid in acute inflammation of the upper respiratory tract. G Clin Med. 1979;60(12):1010-1019. Italian.

34. Wintenberger C, Boccon-Gibod I, Launay D, et al. Tranexamic acid as maintenance treatment for non-histaminergic angioedema: analysis of efficacy and safety in 37 patients. Clin Exp Immunol. 2014; 178(1):112-117.

35. Sheffer AL, Austen KF, Rosen FS. Tranexamic acid therapy in hereditary angioneurotic edema. $N$ Engl J Med. 1972;287(9):452-454.

36. Ker K, Edwards P, Perel P, Shakur H, Roberts I. Effect of tranexamic acid on surgical bleeding: systematic review and cumulative metaanalysis. BMJ. 2012;344:e3054.

37. Ross J, Al-Shahi Salman R. The frequency of thrombotic events among adults given antifibrinolytic drugs for spontaneous bleeding: systematic review and meta-analysis of observational studies and randomized trials. Curr Drug Saf. 2012;7(1):44-54.

38. Pandolfi M, Holmberg L, Turesson I. Coagulation and platelet adhesion-inducing factor in the endothelium of the retinal vessels. $\mathrm{Am}$ J Ophthalmol. 1975;80(1):47-50.

39. Ashton N. Vascular basement membrane changes in diabetic retinopathy. Montgomery lecture, 1973. Br J Ophthalmol. 1974;58(4): 344-366.

40. Green WR, Chan CC, Hutchins GM, Terry JM. Central retinal vein occlusion: a prospective histopathologic study of 29 eyes in 28 cases. Trans Am Ophthalmol Soc. 1981;79(1):371-422.

41. Sperduto RD, Hiller R, Chew E, et al. Risk factors for hemiretinal vein occlusion: comparison with risk factors for central and branch retinal vein occlusion: the eye disease case-control study. Ophthalmology. 1998; 105(5):765-771.

42. Berntorp E, Follrud C, Lethagen S. No increased risk of venous thrombosis in women taking tranexamic acid. Thromb Haemost. 2001; 86(2):714-715.

43. Yang B, Li H, Wang D, He X, Zhang C, Yang P. Systematic review and meta-analysis of perioperative intravenous tranexamic acid use in spinal surgery. PLoS One. 2013;8:e55436.

44. CRASH-2 collaborators, Roberts I, Shakur H, et al. The importance of early treatment with tranexamic acid in bleeding trauma patients: an exploratory analysis of the CRASH-2 randomised controlled trial. Lancet. 2011;377(9771):1096-1101.

45. Godier A, Roberts I, Hunt BJ. Tranexamic acid: less bleeding and less thrombosis? Crit Care. 2012;16(3):135.

46. Shimura M, Yasuda K, Yasuda M, Nakazawa T. Visual outcome after intravitreal bevacizumab depends on the optical coherence tomographic patterns of patients with diffuse diabetic macular edema. Retina. 2013; 33(4):740-747.

47. Kang HM, Chung EJ, Kim YM, Koh HJ. Spectral-domain optical coherence tomography (SD-OCT) patterns and response to intravitreal bevacizumab therapy in macular edema associated with branch retinal vein occlusion. Graefes Arch Clin Exp Ophthalmol. 2013;251(2): 501-508.

48. Ohashi H, Oh H, Nishiwaki H, Nonaka A, Takagi H. Delayed absorption of macular edema accompanying serous retinal detachment after grid laser treatment in patients with branch retinal vein occlusion. Ophthalmology. 2004;111(11):2050-2056.

49. Abri Aghdam K, Reznicek L, Soltan Sanjari M, et al. Peripheral retinal non-perfusion and treatment response in branch retinal vein occlusion. Int J Ophthalmol. 2016;9(6):858-862. 
Clinical Ophthalmology

\section{Publish your work in this journal}

Clinical Ophthalmology is an international, peer-reviewed journal covering all subspecialties within ophthalmology. Key topics include: Optometry; Visual science; Pharmacology and drug therapy in eye diseases; Basic Sciences; Primary and Secondary eye care; Patien Safety and Quality of Care Improvements. This journal is indexed on

PubMed Central and CAS, and is the official journal of The Society of Clinical Ophthalmology (SCO). The manuscript management system is completely online and includes a very quick and fair peer-review system, which is all easy to use. Visit http://www.dovepress.com/ testimonials.php to read real quotes from published authors. 